\title{
Generalized regression and feed-forward back propagation neural networks in modelling porosity from geophysical well logs
}

\author{
Ahmed Amara Konaté • Heping Pan • \\ Nasir Khan · Jie Huai Yang
}

Received: 27 December 2013/Accepted: 21 August 2014/Published online: 11 September 2014

(C) The Author(s) 2014. This article is published with open access at Springerlink.com

\begin{abstract}
Geophysical formation evaluation plays a fundamental role in hydrocarbon exploration and production processes. It is a process which describes different reservoir parameters using well field data. Porosity is one of the parameters that determines the amount of oil present in a rock formation and research in this area is mainly carried out by engineers and geoscientists in the petroleum industry. Accurate prediction of porosity is a difficult problem. This is mostly due to the failure in the understanding of spatial porosity parameter distribution. Artificial neural networks have proved to be a powerful tool for mapping complicated and non-linear relationships in petroleum studies. In this study, we analyze and compare generalized regression neural network (GRNN) and feedforward back propagation neural network (FFBP) in modeling porosity in Zhenjing oilfield data. This study is calibrated on four wells of Zhenjing oilfield data. One well was used to find an empirical relationship between the well $\operatorname{logs}$ and porosity, while the other three wells were used to test the model's predictive ability in the field, respectively. The findings proved that the GRN network can make more accurate and credible porosity parameter estimation than the commonly used FFBP network. Artificial intelligence can be exploited as a powerful instrument for predicting reservoir properties in geophysical formation evaluation and reservoir engineering in petroleum industry.
\end{abstract}

A. A. Konaté · H. Pan $(\bowtie) \cdot$ N. Khan · J. H. Yang

Institute of Geophysics and Geomatic, China University

of Geosciences, Wuhan 430074, Hubei Province, China

e-mail: panpinge@163.com

A. A. Konaté

e-mail: konate77@yahoo.fr
Keywords Porosity prediction - Generalized regression neural network Feed-forward back propagation . Computational geophysics - Reservoir evaluation · Reservoir properties · Geophysical well logs

\section{Introduction}

A major activity in evaluating reservoir is examining the impact of reservoir heterogeneities on reservoir behavior. Heterogeneity in evaluating reservoir is referred to as nonlinear and non-uniform spatial distribution of rock properties such as porosity, permeability and fluids (oil, gas, water) saturation (Mohaghegh et al. 1996). However, it is difficult to predict rock properties due to the form and spatial distribution of these heterogeneities, also the applicability of traditional analytical techniques such as multivariate regression are limited in this context. Several authors such as Mohaghegh et al. (1996) and Handhel (2009) in their related researches buttress these complexities for predicting in heterogeneity reservoir in oil and natural gas field studies.

Understanding the form and spatial distribution of rock properties is fundamental to a successful characterization of petroleum reservoirs (Haldorsen and Damsleth 1993; Wong et al. 1995). In this prevalent situation, it is useful to construct a model that understands rock properties and has the capabilities to make a good prediction. To build a model for predicting requires a set of mathematical equations which describe the dynamic behavior of the process, in other words link a number of input variables with a set of results.

This is a typical problem that can be solved by artificial neural network (ANN) if the phenomenon to be modeled is non-linear; such as the one used in this research. ANNs

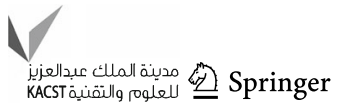


(Hecht-Nielsen 1989) offer an alternative that has the potential to establish a model from non-linear, complex and multidimensional data. They usually take little time to predict output response for any input value that falls in the range of the training data.

Artificial neural network offers real benefit over traditional modeling, including the ability to handle large amounts of noisy data from dynamic and nonlinear systems without a priori information of the processes involved, ANN provides an adequate solution even when the data are incomplete or ambiguous (Handhel 2009). One of the interesting properties of ANN is that it makes accurate predictions. This predictive ability is that it has a degree of liberty that allows it to better capture the non-linearity of a system compared to other modeling techniques.

Artificial neural network has become progressively popular in the petroleum industry and has been widely applied in many petroleum industry applications (see review in van der Baan and Jutten 1992; Poulton 2002). In most of these publications, the feed-forward back propagation neural network (FFBP) configuration is proposed. However, despite the popular applications of FFBP as shown by these publications, the FFBP suffers mostly from the weight initialization randomly given, and the local minima problems, which can often lead the model to evolve in an inaccurate direction. More precisely there are chances that the model never converges to the optimal solution. Furthermore, FFBP training time is often slow and in network architecture optimization, FFBP still have too much human interferences, i.e. the determination of the hidden layer and the number of hidden layer neurons still depend on the user. An alternative way to avoid the above problem is to employ generalized regression neural network (GRNN). As mentioned by Specht (1991), in GRN network optimization process only one parameter (smoothness parameter) has to be adjusted in one pass through the data; no iterative procedure is required; the estimate is confined by the minimum and maximum of the data; convergence guaranteed; fast and stable.

Based on the aforementioned assertions, the researchers were motivated to propose GRNN to predict porosity using four geophysical well logs. The prediction performances were quantified, and compared to FFBP.

Porosity is one of the key petrophysical parameters in evaluating reservoirs to optimize the production of oil and natural gas fields. It is one of the factors that determines the amount of oil present in a rock formation and research in this area is mostly carried out by engineers and geoscientists in the petroleum industry.

This study focuses on developing a model based on ANN that is applicable to predict porosity at Zhenjing oilfield China. It highlights the comparison between GRNN over FFBP in porosity modeling and estimation on common testing set. It also as well as examines the model's ability to predict porosity in the oilfield.

The findings showed that, GRNN compared to FFBP, GRNN model for modeling porosity using geophysical well $\operatorname{logs}$ is significant for the geophysical exploration undertaken in the petroleum industry.

\section{Database}

Four wells named Well\#A, Well\#B, Well\#C and Well\#D from Zhenjing oilfield China were used to provide physicals $\log$ and core porosity data. The physicals logs consisted of bulk density (DEN), compensated neutron porosity (CNL), acoustic (AC) and deep induction resistivity (ILD). The DEN, CNL and $\mathrm{AC}$ respond to the characteristics of rock directly adjacent to the borehole. A combination of these logs provides more accurate estimations of porosity. These geophysical logs are also known as porosity logs. The difference existing between these porosity logs is that, the DEN and CNL are nuclear measurements while AC uses acoustic measurements. However, ILD is an electric log that measures the resistivity of the un-invaded zone of the formation. A crucial use of ILD is the determination of hydrocarbon contained within the pore space of the formations traversed by the well. Figure 1 shows the geophysical well logs used in this study.

Logging tool responses are badly affected by breakout of wall-rock during drilling, as well as stick-and-pull as logging tools are winched up the well (Yan 2002). Keeping this in mind, during this study, the data set from the three wells were carefully examined. All geophysical well logs which exhibited strange, and possibly inappropriate data were ignored. In addition, correction of the offset between core depth and logging depth was done, so that the geophysical well logs and experimental data may be matched and integrated effectively.

The data from Well\#A (1046 core and log data) were chosen to provide the training patterns. This well was chosen because, it had the most complete set of core and log data. It was randomly divided into training data (70 \%) and testing data $(30 \%)$. The data from Well\#B (152 core and $\log$ data), Well\#C (91 core and $\log$ data) and Well\#D (40 core and log data) were used to test the model's ability to predict porosity in the oilfield.

\section{Methods}

Artificial neural network is a set of computing systems that imitates biological processes through the use of interconnections between simple artificial neurons. While the 


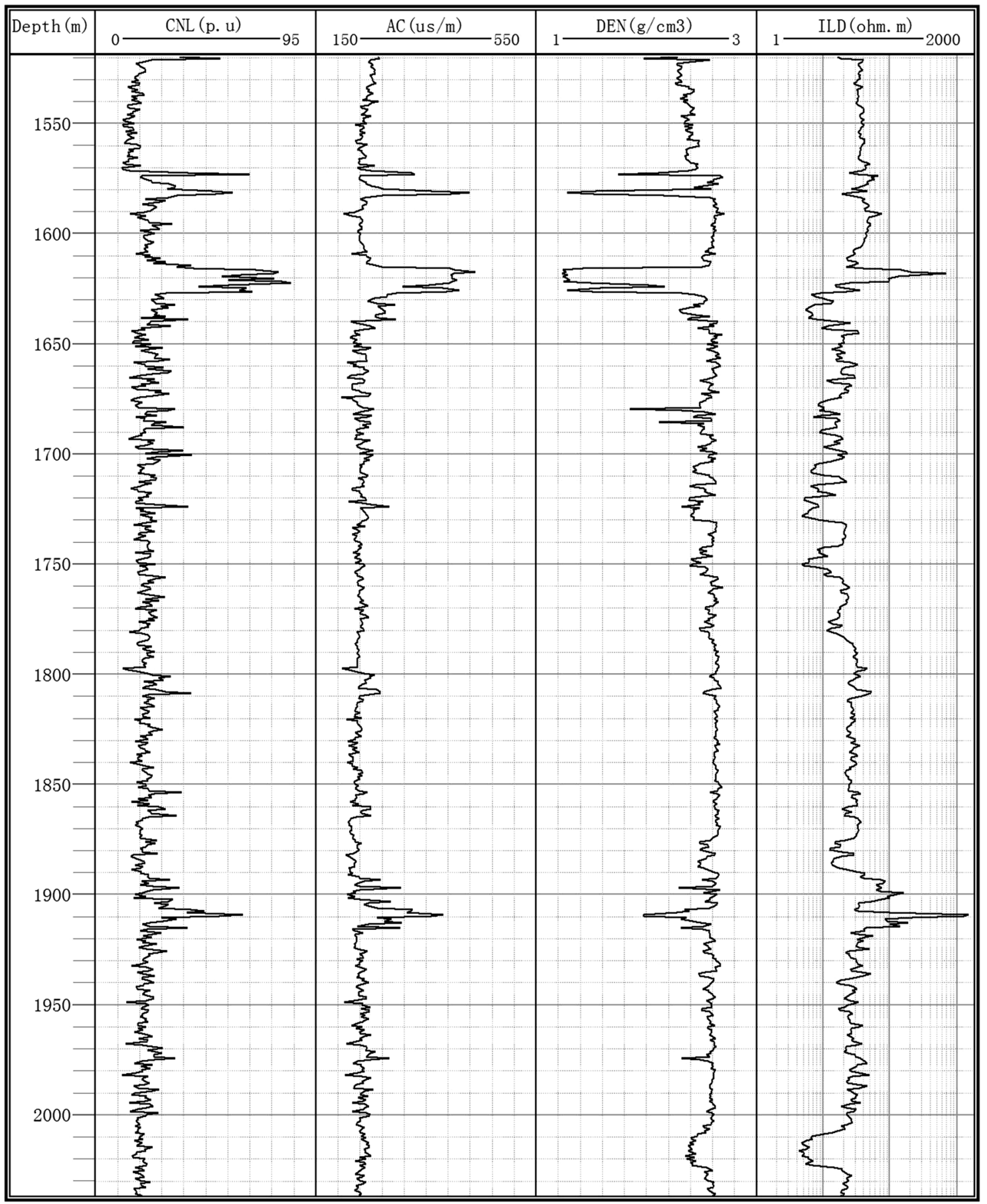

Fig. 1 Geophysical well logs used in this study. Well\#A

concept may seem to belong to recent technological developments, it has been discussed long before the current trend in computers with the objective of trying to duplicate the learning abilities of biological neurons that constitute the basic element of the brain. From a technical point of view, each neuron is connected to others by direct links. Each link is associated with a weight which represents the information used by the network to solve the problem.
An artificial neuron is a calculating unit that receives a certain number of inputs directly from the environment or from upstream neurons. When the information comes from a neuron, it is associated with a weight $(w)$, which represents the ability of the upstream neuron to excite or inhibit downstream neurons. Each neuron is provided with a unique output, which then branches out to supply a variable number of downstream neurons. 


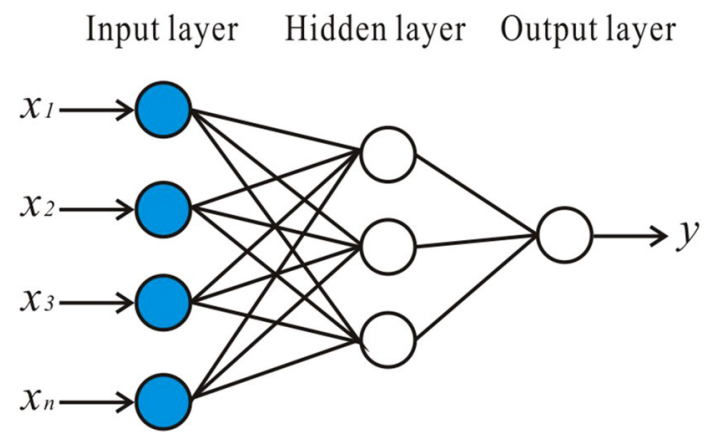

Fig. 2 Schematic representation of FFBP

Artificial neural network presumes that the true underlying function that governs the relationship between inputs and outputs is not known a priori. It determines a mathematical function which can properly approach the representation of inputs and outputs.

One of the major aspects of ANN is the training process, which can be either supervised or unsupervised. In this study, the former was used for prediction approach. It is the most widely applied in geophysical fields (van der Baan and Jutten 1992; Poulton 2002). Supervised learning, i.e. guided learning by "teacher"; requires a training set which consists of input vectors and a target vector associated with each input vector. The advantage of supervised training is that the output can be interpreted based on the training values. The disadvantage is that a large number of inputs and outputs are required to guarantee adequate training. In this study, the given training dataset (1046 core and log data) is sufficient and requires a supervised learning model.

\section{Feed-forward back propagation neural network (FFBP)}

Feed-forward back propagation neural network is one of the most popular ANN models for engineering applications (Haykin 2007). The FFBP represented in Fig. 2 comprises of three layers; the input layer receiving the information on the neurons represented by circles and an output layer having a single neuron and giving the internal calculation result. Between these two layers, there is another layer not visible from the outside called the hidden layer responsible for performing intermediate computations.

Determination of the number of hidden layers, hidden neuron and type of transfer function plays an important role in FFBP model constructions (White 1992). The number of hidden layers required depends on the complexity of the relationship between the input and the target parameters. It has an impact on the quality of the learning, FFBP comprising more hidden layers are very rare, given that each new layer increases the quantity of calculations. In majority problems only one hidden layer is sufficient. Hornik et al. (1989) proved that FFBP with one hidden layer is enough to approximate any continuous function. Therefore, one hidden layer was employed in the current research. Besides, transfer functions for the hidden nodes are needed to introduce non-linearity into the network. In this study, the sigmoid was selected as activation function of the hidden neurons while a linear activation function was used in the output neurons.

Next, the choice of the optimal number of hidden layer neurons is an essential decision in the modeling phase. If an insufficient number of neurons are used, the network will be unable to model complicated data, and the resulting fit will be poor. Many hidden neurons will ensure correct training, and the network will be able to appropriately predict the data it has been trained on, but its performance on new data and its ability to generalize will be compromised (Abraham 2005). Whereas, with very few hidden neurons, the network may be inept to learn the associations between the input and output variables. In this sense, the error will fail to fall below an adequate level (Abraham 2005). Thus, a compromise has to be reached between too many and too few neurons in the hidden layer. In this study, the optimal number in hidden layer was selected by experimental trial based on the smallest mean square error (MSE).

The objective of training the FFBP is to find optimal connection weights $\left(w^{*}\right)$ in such a manner that the value of calculated outputs for each example matches the value of desired outputs. This is typically a non-linear optimization problem, where $w^{*}$ is given by Eq. (1)

$w^{*}=\arg \min E(w)$

where $w$ is weight matrix and $E(w)$ is an objective function on $w$, which is to be minimized.

The $E(w)$ is evaluated at any point of $w$ given by Eq. (2)

$E(w)=\sum_{p} E_{p}(w)$

$p$ is the number of examples in the training set and $E_{p}(w)$ is the output error for each example $p . E_{p}(w)$ is expressed by Eq. (3)

$E_{p}(w)=\frac{1}{2} \sum_{j}\left(d_{p j}-y_{p j}(w)\right)^{2}$

where $y_{p j}(w)$ and $d_{p j}$ are the calculated and desired network outputs of the $j$ th output neuron for $p$ th example, respectively. The objective function to be minimized is represented by Eq. (4):

$E(w)=\frac{1}{2} \sum_{p} \sum_{j}\left(d_{p j}-y_{p j}(w)\right)^{2}$.

For each learning (training) process, the network calculated output value is compared to the desired output value. If there is a difference between the calculated and desired output network, the synaptic weights which 
contribute to generate a significant error will be changed more significantly than the weight that led to a marginal error. The adaptation of the weights begins at the output neurons and then continues toward the input data. There are many algorithms available to perform this weight selection and adjustment (see Bishop 1995). One of the most popular is the gradient descent, which suffers from slow convergence times and can easily get trapped in local minima within the vector space of $w$ during the learning process; this leads the model to evolve in an accurate direction. In this research, this algorithm was applied with no guarantee in obtaining the optimal trained network for given data. Therefore, LevenbergMarquardt algorithm (LMA) was chosen to train the neural network. LMA is considered one of the most efficient training algorithms; the study of Hagan and Menhaj (1994) proved that LMA is faster and has more stable convergence as compared to gradient descent algorithm.

\section{Generalized regression neural network (GRNN)}

Generalized regression neural network is related to the radial basis neural networks, which are found on kernel regression. It can be treated as a normalized radial basis neural networks in which there is a hidden neuron centered at every training case. These radial basis function units are generally probability density function such as the Gaussian (Celikoglu 2006). The use of a probability density function is particularly gainful due to its ability to converge to the underlying function of the data with only limited training data available. In GRNN optimization process only one parameter (smoothing) has to be adjusted in one pass through the data; no iterative procedure is required; the estimate is confined by the minimum and maximum of the data. Furthermore, GRNN approximates any arbitrary function between input and target vectors; fast training and convergence to the optimal regression surface as the training data becomes very large (Specht 1991). This makes GRNN a very advantageous tool to perform predictions.

Figure 3 is a representation of the GRNN architecture with four layers: an input layer, a hidden layer, a summation layer, and an output layer. As it can be seen in Fig. 3, the input layer is completely linked to the hidden layer called

Input layer Pattern layer Summation layer Output layer

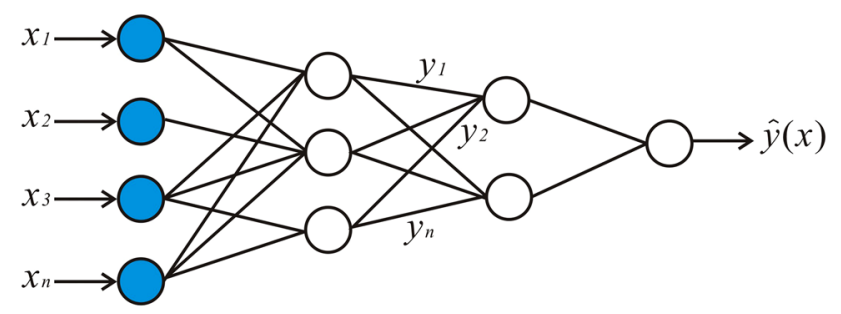

Fig. 3 Schematic diagram of a GRNN architecture "pattern layer". In the pattern layer, each neuron is a training pattern and its output represents a measure of the distance between the input and the stored patterns. The hidden layer is fully linked to the third layer, called "summation layer". This later has two different types of summation: S-summation neuron (summation units) and D-summation neuron (a single division unit). S-summation neuron determines the sum of the weighted outputs of the hidden layer, whereas the D-summation neuron determines the unweighted outputs of the pattern neurons. As also mentioned in Fig. 3, the synaptic weight between a neuron in the hidden layer and an S-summation layer neuron is the target output value corresponding $\left(y_{i}\right)$. The summation layer and the last layer of the network, "output layer" together execute a normalization of output set. In the training of the network, radial basis function (Gaussian) and linear transfer functions are used in hidden and output layers, respectively.

In reference to Specht (1991), let us suppose that $f(x, y)$ represents the known joint continuous probability density function of a vector random variable, $x$, and a scalar random variable, $y$. The regression of $y$ on $x$ is expressed by Eq. (5):

$E[y / x]=\frac{\int_{-\infty}^{\infty} y f(x, y) d y}{\int_{-\infty}^{\infty} f(x, y) d y}$.

If the density $f(x, y)$ is unknown, it must generally be predicted (estimated) from a sample of observations of $x$ and $y$. The probability estimator $\hat{f}(x, y)$ given in Eq. (6), is based upon sample values of the variables $x$ and $y$ represented by $x^{i}$ and $y^{i}$, respectively. $n$ and $p$ represent the number of sample observations and the dimension of the vector variable $x$, respectively:

$$
\begin{aligned}
\hat{f}(x, y)= & \frac{1}{(2 \pi)^{(p+1) / 2} \sigma^{(p+1)}} \\
& \times \frac{1}{n} \sum_{i=1}^{n} \exp \left[-\frac{\left(x-x^{i}\right)^{T}\left(x-x^{i}\right)}{2 \sigma^{2}}\right] \exp \left[-\frac{\left(y-y^{i}\right)^{2}}{2 \sigma^{2}}\right] .
\end{aligned}
$$

A meaningful explanation of the probability estimate $\hat{f}(x, y)$ is that, it allocates sample probability of smoothness parameter $(\sigma)$ for each sample $x^{i}$ and $y^{i}$, and the probability estimate is the sum of those sample probabilities.

When defining the scalar function given by Eq. (7)

$D_{i}^{2}=\left(x-x^{i}\right)^{T}\left(x-x^{i}\right)$.

Therefore, a prediction performed by GRNN, $\hat{y}(x)$ to an unknown input vector $x$ is expressed in Eq. (8)

$$
\hat{y}(x)=\frac{\sum_{i=1}^{n} y^{i} \exp \left(-\frac{D_{i}^{2}}{2 \sigma^{2}}\right)}{\sum_{i=1}^{n} \exp \left(-\frac{D_{i}^{2}}{2 \sigma^{2}}\right)}
$$


Table 1 Pearson correlation $(r)$ of observed porosity versus geophysical well logs data (Well\#A)

\begin{tabular}{lrll}
\hline $\begin{array}{l}\text { Pearson } \\
\text { correlation }(r)\end{array}$ & $r$ value & $\begin{array}{l}r \text { probability } \\
(p)\end{array}$ & $\begin{array}{l}\text { Level of } \\
\text { significance }\end{array}$ \\
\hline Porosity vs ILD & $-0.42^{*}$ & 0.000 & 0.01 \\
Porosity vs AC & $0.83^{*}$ & 0.000 & 0.01 \\
Porosity vs DEN & $-0.60^{*}$ & 0.000 & 0.01 \\
Porosity vs CNL & $0.51^{*}$ & 0.000 & 0.01 \\
\hline
\end{tabular}

* Correlation statistically significant at the $0.01(p \leq 0.01)$

where each sample, $x^{i}$ of $x$ is used as the mean of a normal distribution.

As mentioned by Specht (1991), the smoothness parameter $\sigma$, is a very important parameter of GRN network. We should keep in mind that, if $\sigma$ is bigger, the predicted (estimated) density is forced to be smooth and in the limit becomes a multivariate Gaussian with covariance $\sigma^{2} I(I=$ unity matrix $)$. In contrary, when $\sigma$ is smaller, the predicted density assumes non Gaussian shapes, but with the hazard that wild points may have a great effect on the estimate. The smoothness parameter $(\sigma)$, is still subject to a search.

\section{Performance criteria}

In diagnostic statistics, there are many ways to quantify the difference between observed values and predicted (estimated) values. In this study, to evaluate FFBP scheme and GRNN scheme, the statistics mean squared error (MSE), coefficient of determination $\left(R^{2}\right)$ and coefficient of correlation $(r)$ were used to quantify performance. They are given by Eqs. (9), (10) and (11), respectively. Where $o_{i}$ is observed porosity value, $\hat{p}_{i}$ is predicted porosity value, $\bar{o}$ is

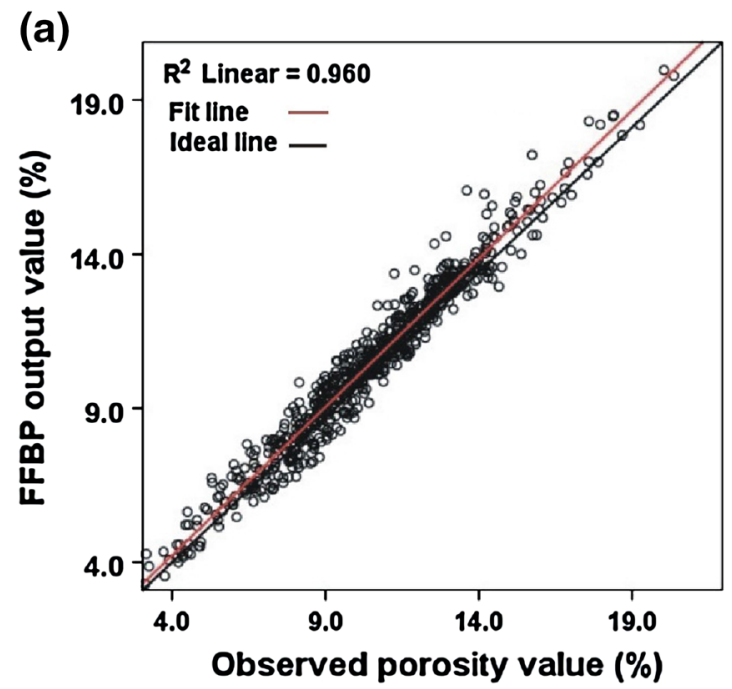

mean observed value; $\bar{p}$ is mean predicted value and $N$ is total number of data

$\mathrm{MSE}=\frac{1}{N} \sum_{i=1}^{N}\left(o_{i}-\hat{p}_{i}\right)^{2}$

MSE, measures the average of the squares of the errors, i.e. the residual errors, which help scientists to understand and interpret the difference between the observed value and estimated values. We should keep in mind that, this indicator measures how near a fit line is to data points. The smaller the MSE, the nearer the fit is to the data points.

$$
R^{2}=\left(\frac{\sum_{i=1}^{N}\left(o_{i}-\bar{o}\right) \times\left(\hat{p}_{i}-\bar{p}\right)}{\sqrt{\sum_{i=1}^{N}\left(o_{i}-\bar{o}\right)^{2} \times \sqrt{\sum_{i=1}^{N}\left(\hat{p}_{i}-\bar{p}\right)^{2}}}}\right)^{2}
$$

This coefficient is a statistical index that expresses the quality of fit estimates of the regression equation and also the intensity of the linear relationship. It helps to have a general idea of the model fit. Its value varies between 0 and 1 , and if the $R^{2}$ value is close to 1 it is sufficient to say that the fit is good.

$$
r=\frac{N \sum_{i=1}^{N} o_{i} \hat{p}_{i}-\sum_{i=1}^{N} o_{i} \sum_{i=1}^{N} \hat{p}_{i}}{\sqrt{\left(N \sum_{i=1}^{N} o_{i}^{2}-\left(\sum_{i=1}^{N} o_{i}\right)^{2}\right)\left(N \sum_{i=1}^{N} \hat{p}_{i}^{2}-\left(\sum_{i=1}^{N} \hat{p}_{i}\right)^{2}\right)}}
$$

$r$ measures the strength of a linear relationship amongst the observed value and estimated variables. In other words, it is an indicator of the scatter around the fit line. If $r$ is close

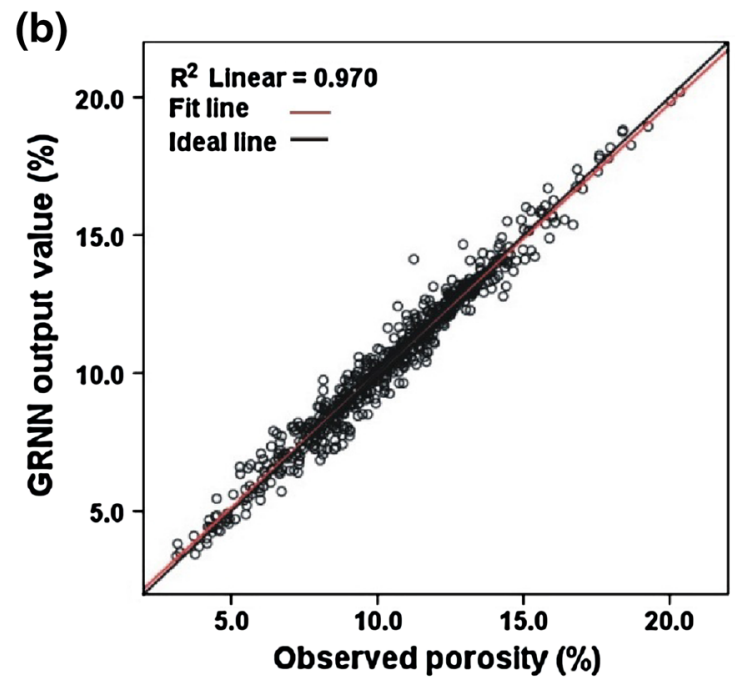

Fig. 4 Cross-plots of predicted porosity against observed porosity. Prediction from FFBP (a) and GRNN (b). Training data Well\#A 
Table 2 Statistical performance of GRNN and FFBP scheme of porosity and observed porosity (Well\#A)

\begin{tabular}{llllllll}
\hline Methods & $\begin{array}{l}\text { Testing data (314 data points) } \\
\text { non trained }\end{array}$ & \multicolumn{3}{l}{$\begin{array}{l}\text { Training data (734 data } \\
\text { point) }\end{array}$} \\
\cline { 2 - 3 } & $R^{2}$ & $r$ & MSE & & $R^{2}$ & $r$ & MSE \\
\hline GRNN & 0.958 & 0.978 & 0.278 & & 0.970 & 0.984 & 0.383 \\
FFBP & 0.940 & 0.969 & 0.381 & & 0.960 & 0.979 & 0.449 \\
\hline
\end{tabular}

to 1 , it means that the relationship between the observed and estimated variables is positive and thereby indicating that the data points (dots) fall nearly along a fit line with positive slope. Whereas, when $r$ is close to -1 , the relationship between the observed and estimated variables is negative and the dots fall nearly along a fit line with negative slope. When $r$ is close to zero, it implies a weak relationship between the observed and estimated variables and that the data points are scattered around the fit line and most of data points are not in good agreement with the fit line.

\section{Results and interpretations}

There are four parameters (geophysical well logs) considered as the inputs for the modeling process. They are bulk density (DEN), compensated neutron porosity (CNL), acoustic (AC) and deep induction resistivity (ILD). Pearson' correlation $(r)$ was used to evaluate the statistical relationship that may exist between each well log and core measured porosity. Table 1 shows the relationship between each well $\log$ and core measured porosity. As can be seen in Table 1, there is significant correlation between each
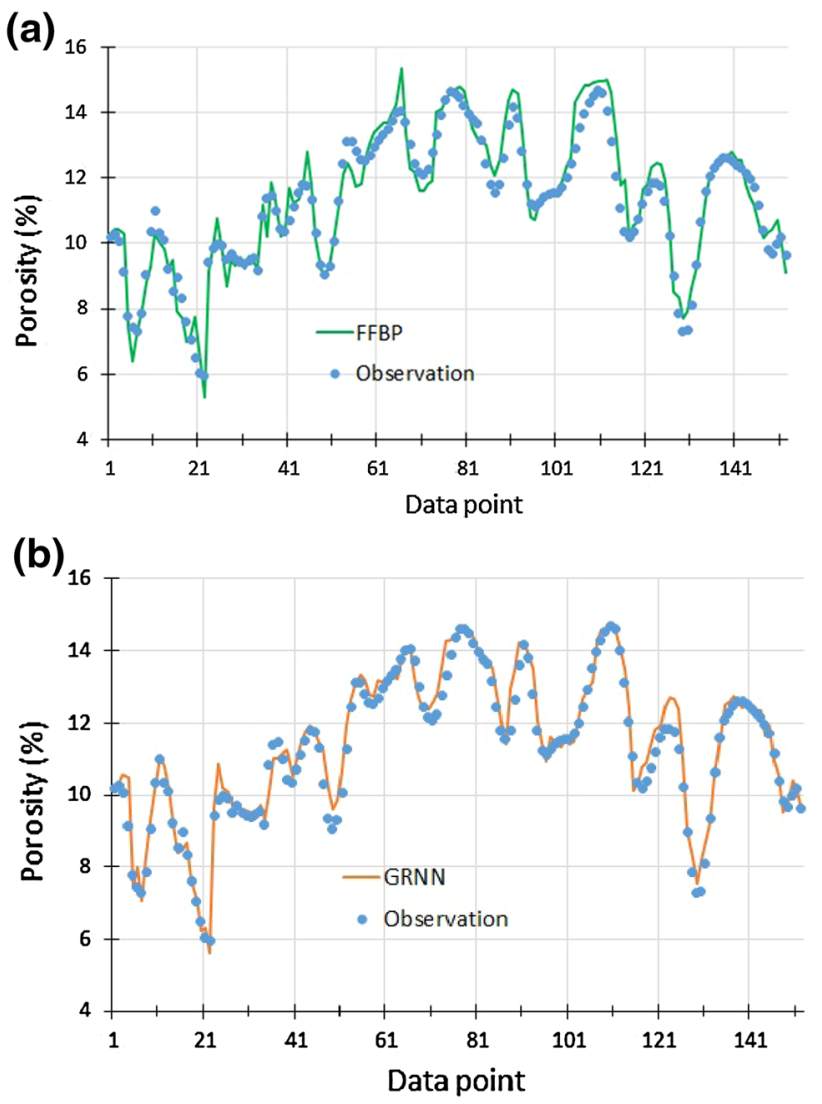

Fig. 6 Prediction results a FFBP, b GRNN. Well\#B

well $\log$ and core measured porosity; with a significant $(p \leq 0.01)$ difference from zero. In other words, the relationship existing between each well log and core measured porosity, respectively, is statistically significant. Statistically significant means that the observed sample dataset
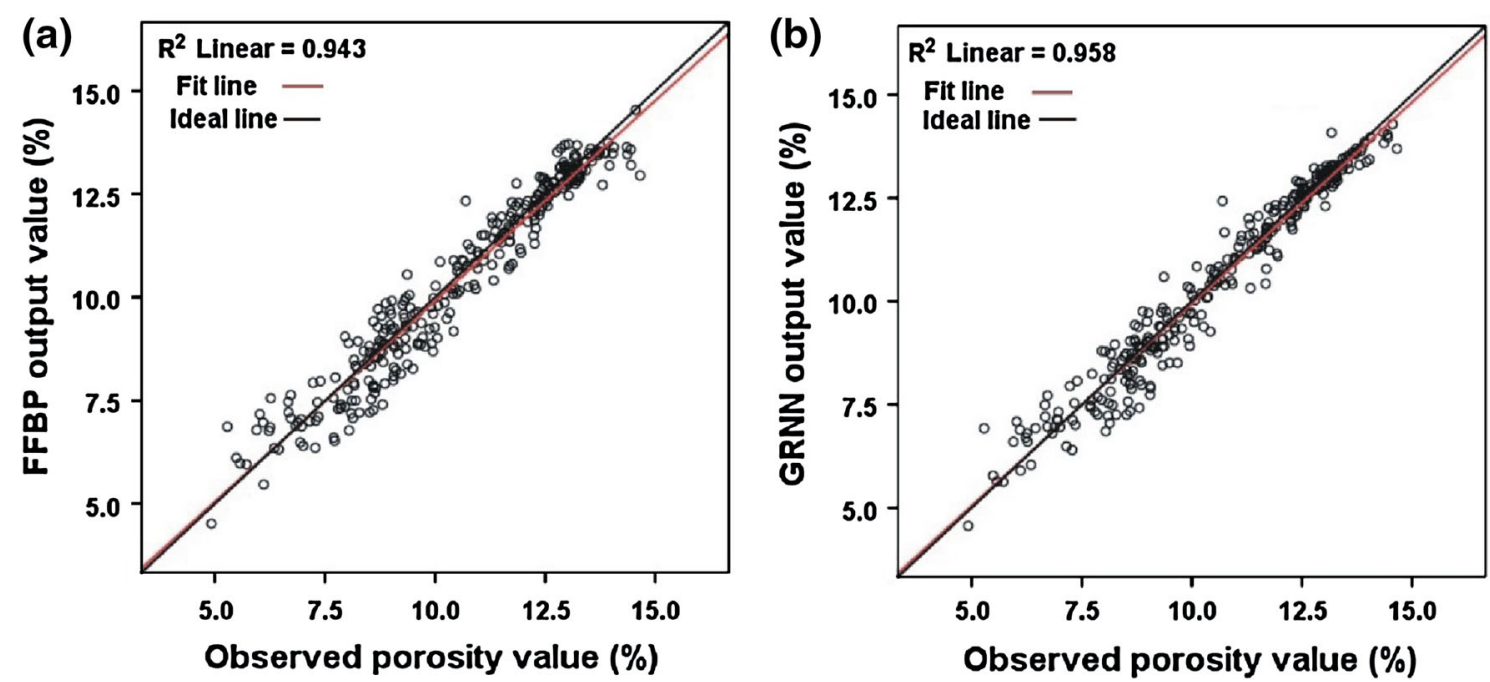

Fig. 5 Cross-plots of predicted porosity against observed porosity. Performance from FFBP (a) and GRNN (b). Testing data Well\#A 

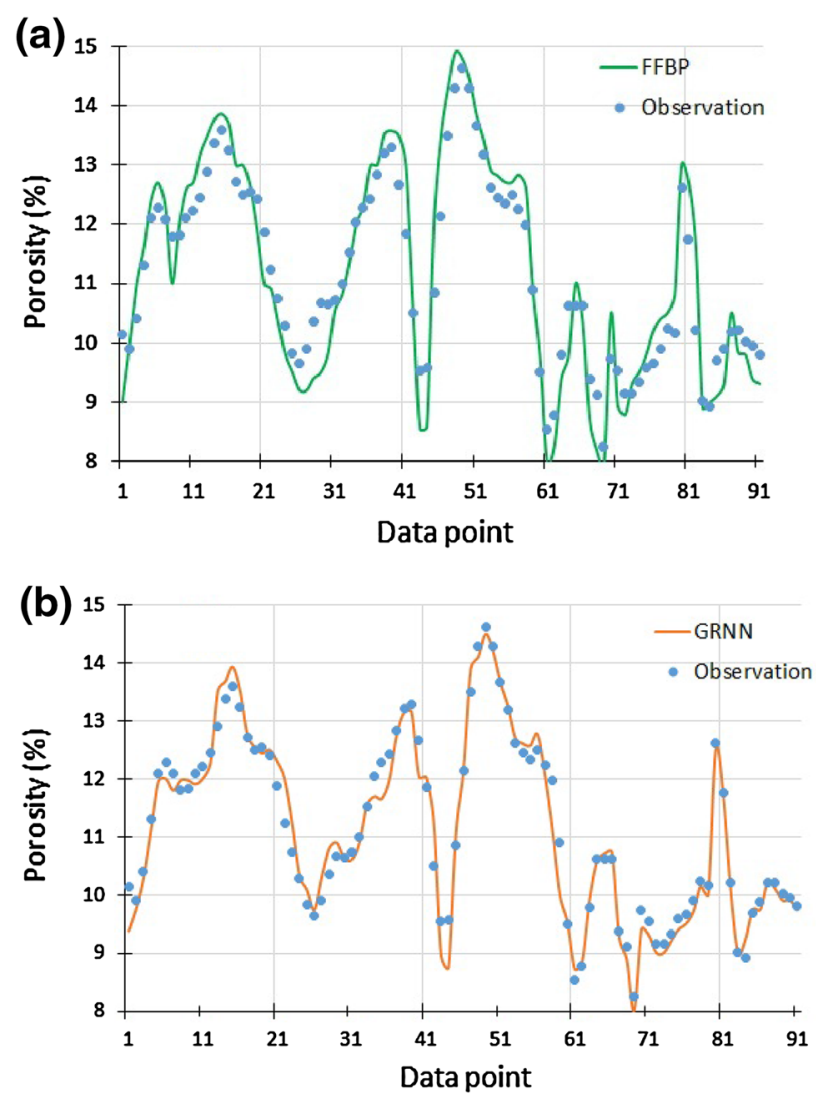

Fig. 7 Prediction results a FFBP, b GRNN. Well\#C

provides ample evidence to reject the null hypothesis that "the population correlation coefficient is zero" $\left(\mathrm{H}_{0}: \rho=0\right)$ thereby concluding that $\rho \neq 0$. Thus, each well $\log$ appears linearly correlated to measured porosity, respectively. Therefore, in this study, Pearson' correlation $(r)$ has confirmed the accuracy of the four geophysical well logs as inputs parameters to the ANNs.

To use the study datasets for ANN training some normalization was performed. The output and all inputs were normalized between 0 and 1 . The data for this study involved different parameters that have dissimilar physical meaning and units. To make sure that each variable is treated similarly in the model, the data were normalized.

The next step was to develop porosity model, integrate core porosity data (target) with well log data (inputs) using ANN algorithms to establish a satisfactory model for the relationship between well log data and rock porosity. The 1043 data points from Well\#A were randomly divided into training data $(70 \%)$ and testing data $(30 \%)$. The training data were used in FFBP and GRNN training. While the testing data (not trained) was used to estimate the prediction ability of the models.

After several trials, the optimal architecture of FFBP was, 4 inputs, one hidden layer of 10 neurons and 1 output.
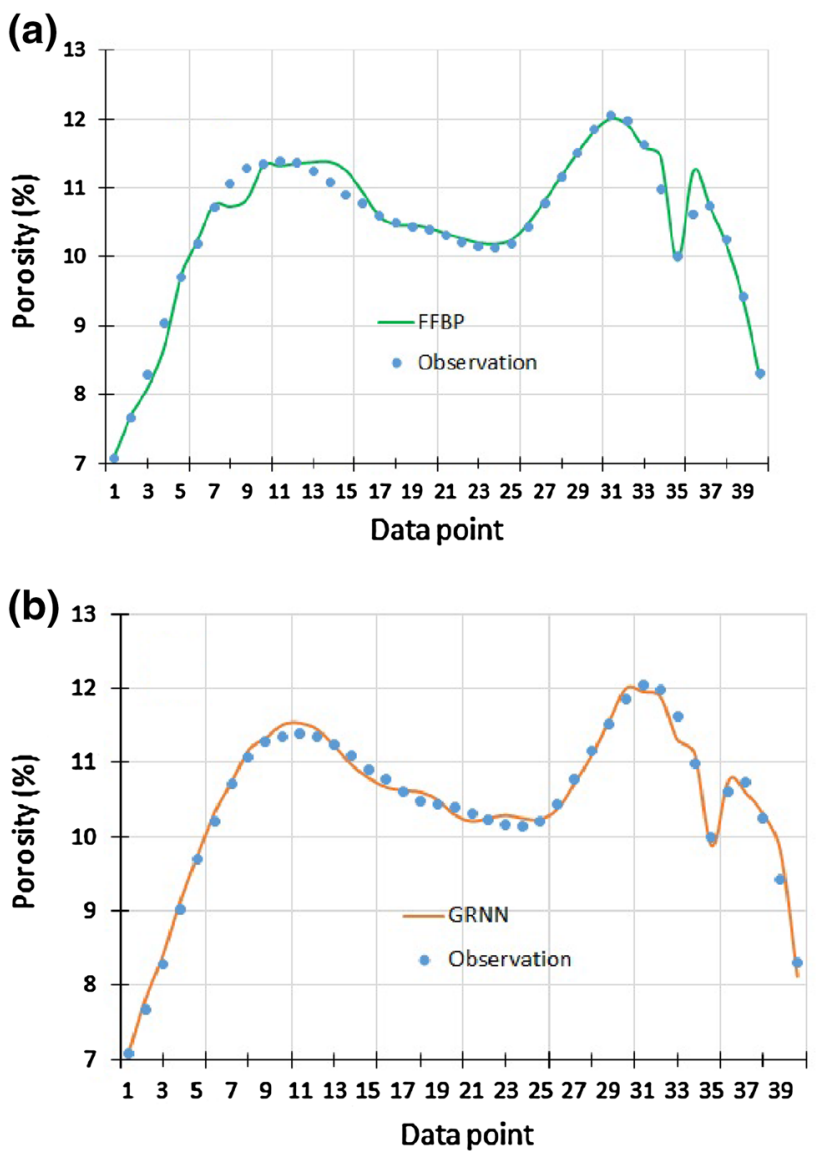

Fig. 8 Prediction results a FFBP, b GRNN. Well\#D

While GRNN structure was 4 inputs, smoothness parameter $(\sigma)=0.02$ and 1 output.

Figure $4 \mathrm{a}, \mathrm{b}$ illustrates cross-plots of predicted porosity against observed porosity for training dataset. From a visual observation of Fig. $4 a$, b, there is a very positive correlation between predicted porosity from the two neural networks scheme and observed porosity, respectively, as shown in the alignment results (dots) obtained by the two neural networks around the lines (fit line and ideal line). This indicates satisfactory training by these networks. However, the neural network in generalized regression structure fit line approaches the ideal line closer than neural network in back propagation structure fit line (Fig. 4a, b). Additionally, the results in Table 2 support the superiority of GRNN training performance, since GRNN structure shows higher $R^{2}$ and $r$ values and lower MSE value, while FFBP structure indicates lower $R^{2}$ and $r$ values and higher MSE value. We can therefore conclude that in this study GRNN model trains (learns) porosity better than FFBP model.

After training step was done, the two neural networks were tested using testing data (not trained) from Well\#A. Figure 5a, b shows cross-plots predicted values versus observed values porosity for testing data (Well\#A). 
Table 3 Statistical performance of GRNN and FFBP scheme of porosity and observed porosity (Well\#B, Well\#C and Well\#D)

\begin{tabular}{|c|c|c|c|c|c|c|c|c|c|}
\hline \multirow[t]{2}{*}{ Methods } & \multicolumn{3}{|c|}{ Well\#B } & \multicolumn{3}{|c|}{ Well\#C } & \multicolumn{3}{|c|}{ Well\#D } \\
\hline & $R^{2}$ & $r$ & MSE & $R^{2}$ & $r$ & MSE & $R^{2}$ & $r$ & MSE \\
\hline GRNN & 0.966 & 0.982 & 0.370 & 0.961 & 0.980 & 0.309 & 0.986 & 0.992 & 0.128 \\
\hline FFBP & 0.936 & 0.967 & 0.544 & 0.922 & 0.960 & 0.519 & 0.973 & 0.984 & 0.188 \\
\hline
\end{tabular}

Analysis of the cross-plot in Fig. 5a, b depicts that the two neural networks fit line, respectively, closely coincides with the ideal line. Additionally, the dots appear alongside the lines (fit line and ideal line). This means that the networks have satisfactorily predicted porosity. A visual check in Table 2, clearly depicts that neural network generalized regression scheme surpasses neural network in back propagation scheme since, GRNN scheme produces higher $R^{2}$ and $r$ values and lower MSE value, while FFBP scheme suffers from lower $R^{2}$ and $r$ values and higher MSE value. We can therefore conclude that in this study GRNN scheme fits porosity better than FFBP scheme.

After successful testing from Well\#A, the two neural networks were also tested using data from Well\#B, Well\#C and Well\#D. Figures 6,7 and 8 visually illustrate the variation of observed porosity values and predicted porosity values from the two models, thus providing a visual exhibition of accuracy. As it can be seen in Figs. 6, 7 and 8 , neural network in generalized regression scheme is in better concordance with observed porosity values as compared to the neural network in back propagation scheme. Table 3 presents the $R^{2}, r$ and MSE statistics for testing data from Well\#B, Well\#C and Well\#D. From Table 3, the results confirm the FFBP scheme weakness as they exhibit lower $R^{2}$ and $r$ values and higher MSE value. This means that the neural network in generalized regression scheme estimate porosity better than the neural network in back propagation scheme.

\section{Summary and conclusions}

The increasing success of ANN application mostly in many techniques can be attributed to its power, adaptability and simplicity. It can be useful to elucidate any complex, nonlinear and dynamic reservoir parameter problems. This is because it does not require a priori information about the functional shape to be estimated.

In the petroleum industry, rock porosity (as well as permeability, lithology) is one of the major concern in reservoir characterization. It is identified during the geophysical exploration phase to estimate the capability of an oilfield and to look for the optimal locations for drilling wells production. Porosity is essential in understanding the crustal heterogeneity of a reservoir. In this light, geophysicists, geologists and engineers are always trying to find a cost effective, fast and robust method for accurate estimation.

In this study innovative effort was made to analyze and compare generalized regression neural network and feedforward back propagation neural network in modeling porosity. The findings indicate that artificial neural network is an appropriate tool for modeling porosity, despite the high degree of heterogeneity of reservoir in Zhenjing oilfield. Additionally, the geophysical well logs (DEN, CNL, $\mathrm{AC}$ and ILD) are significant parameters to be considered for developing a porosity model.

From all the results in this study we see that, neural network generalized regression scheme is better than neural network in back propagation scheme. This obviously indicates GRNN model outperforms FFBP model. This assertion has also been echoed by several authors such as Specht (1991); Cigizoglua and Alp (2006); and Sun et al. (2008) in their respective research areas. These researchers, have mentioned promising advantages of GRNN model over FFBP model.

In conclusion GRNN gives better prediction accuracy in predicting porosity than FFBP. The GRNN exhibited better precision with the core porosity data. Due to it great flexibility and capability in dealing with non-linear problem in actual situation, GRN network scheme can thus serve as a cost effective approach for the petroleum industry by way of reducing the necessity of coring because, it may allow improved prediction in uncored intervals. Furthermore, this method may be a very useful tool in aiding prediction of future wells.

However, artificial neural network still has some limitations. For example, in network construction and adjusting of learning parameters, there are too many human interferences. Nevertheless, all these problems are now being investigated which can be expected to provide satisfactory answers for future use.

Open Access This article is distributed under the terms of the Creative Commons Attribution License which permits any use, distribution, and reproduction in any medium, provided the original author(s) and the source are credited. 


\section{References}

Abraham A (2005) Artificial neural networks, handbook of measuring system design. In: Sydenham PH, Thorn R (eds) ISBN: 0-47002143-8, Wiley, Hoboken

Bishop C (1995) Neural networks for pattern recognition. Oxford Press, Oxford

Celikoglu HB (2006) Application of radial basis function and generalized regression neural networks in non-linear utility function specification for travel mode choice modelling. Math Comput Model 44:640-658

Cigizoglua HK, Alp M (2006) Generalized regression neural network in modelling river sediment yield. Adv Eng Softw 37:63-68

Hagan MT, Menhaj MB (1994) Training feed forward techniques with the Marquardt algorithm. IEEE Trans Neural Netw 5(6):989-993

Haldorsen HH, Damsleth E (1993) Challenges in reservoir characterization. AAPG Bull 77:541-551

Handhel AM (2009) Prediction of reservoir permeability from wire logs data using artificial neural networks. Iraqi J Sci 50(1):67-74

Haykin S (2007) Neural networks: a comprehensive foundation, Third edn. Prentice Hall, Inc., Upper Saddle River

Hecht-Nielsen R (1989) Theory of backpropagation neural networks. In: Presented at IEEE Proceedings, international conference on neural network, Washington DC
Hornik K, Stinchcombe MB, White H (1989) Multilayer feed forward networks are universal approximators. Neural Netw 2(5):359-366

Mohaghegh S, Arefi R, Ameri S, Aminian K, Nutter R (1996) Petroleum reservoir characterization with the aid of artificial neural networks. J Petrol Sci Eng 16(4):263-274

Poulton MM (2002) Neural networks as an intelligence amplification tool: a review of applications. Geophysics 67(3):979-993

Specht DF (1991) A general regression neural network. IEEE Trans Neural Netw 2(6):568-576

Sun G, Hoff SJ, Zelle BC, Nelson MA (2008) Nelson Development and comparaison of backpropagation and generalized neural network models to predict diurnal and seasonal gas and PM10 concentrations and emissions from buildings. Trans ASABE 51(2):685-694

Van der Baan M, Jutten C (1992) Neural networks in geophysical applications. Geophysics 65(4):1032-1047

White H (1992) Artificial neural networks. Approximation and learning theory. Blackwell, Cambridge

Wong PM, Gedeon TD, Taggart J (1995) An improved technique in porosity prediction: a neural network approach. IEEE-TGRS 33(4):971-980

Yan J (2002) Reservoir parameters estimation from well log and core data: a case study from the North Sea. Pet Geosci 8:63-69 